\title{
Percepción de los docentes sobre las culturas institucionales, políticas y prácticas inclusivas. Estudio de caso de la Universidad de Cuenca-Ecuador
}

\author{
Teachers' perception of the institutional cultures, politics and inclusive practices. \\ Case-study the University of Cuenca-Ecuador
}

\author{
Ruth Clavijo-Castillo ${ }^{1 *}$ (D), María-Josefa Bautista-Cerro ${ }^{2}$ (D) \\ ${ }^{1}$ Facultad de Psicología, Universidad de Cuenca, Avenida 12 de Abril y Loja, Cuenca, Ecuador. \\ ${ }^{2}$ Facultad de Educación, Universidad Nacional de Educación a Distancia (UNED), Madrid, España. \\ *Autor de correspondencia: ruth.clavijo@ucuenca.edu.ec \\ Fecha de recepción: 15 de octubre de 2021 - Fecha de aceptación: 9 de noviembre de 2021
}

\begin{abstract}
RESUMEN
El derecho a la educación es un derecho humano fundamental y, según la UNESCO (2015), la educación inclusiva y de calidad está basada en la equidad, la flexibilidad y la adaptabilidad, todo ello con el objetivo de no dejar a nadie atrás. En Ecuador, la educación inclusiva es un proceso reciente y sufrió una serie de cambios influenciados por políticas y regulaciones que abordaron la diversidad y garantizaron el derecho a la educación superior para todos los estudiantes. Este artículo presenta los resultados de una investigación diseñada para evaluar la creencia de los docentes de la Universidad de Cuenca (Ecuador) sobre culturas, políticas y prácticas inclusivas, utilizando como instrumento el Index for inclusion. Las dimensiones evaluadas del índice son la creación de culturas, la elaboración de políticas y el desarrollo de prácticas inclusivas. Todas las dimensiones examinadas muestran un nivel regular de desempeño; sin embargo, existe una distinción entre la creación de culturas inclusivas en un grado que se asemeja mucho al desarrollo de prácticas inclusivas, mientras que la dimensión de la formulación de políticas inclusivas recibió la percepción más baja. Los hallazgos permiten diferenciar la posición de los docentes sobre los aspectos analizados de la educación inclusiva y resaltar los cuellos de botella que se abordarán en el futuro, especialmente la formación del profesorado en educación inclusiva.
\end{abstract}

Palabras clave: Educación inclusiva, inclusión, index for inclusion, universidad, docentes.

\begin{abstract}
The right to education is a fundamental human right, and according to UNESCO (2015) is inclusive and quality education based on equity, flexibility and adaptability, all with the aim of leaving no one behind. In Ecuador, inclusive education is a recent process and underwent a series of changes influenced by policies and regulations that addressed diversity and guaranteed the right to higher education for all students. This article presents the results of an investigation designed to assess the belief of the teachers at the University of Cuenca (Ecuador) about inclusive cultures, policies and practices, using the Index for Inclusion as instrument. The evaluated dimensions of the index are the creation of cultures, the elaboration of policies, and the development of inclusive practices. All examined dimensions show a regular level of performance, however a distinction exists between the creation of inclusive cultures to a degree that closely resembles the development of inclusive practices, while the dimension of inclusive policymaking received the lowest feeling. The findings allow to differentiate the position of teachers on the analyzed aspects of inclusive education and highlight the bottlenecks to be addressed in the future, especially teacher training in inclusive education.
\end{abstract}

Keywords: Inclusive education, inclusion, index for inclusion, university, professors.

\section{INTRODUCCIÓN}

La Agenda 2030 de Naciones Unidas presenta como uno de sus compromisos específicos poner fin a la discriminación y la exclusión, reduciendo las desigualdades y vulnerabilidades que ponen en riesgo el potencial de los individuos y de la humanidad en su conjunto. El ODS4 de esta Agenda se centra en "Garantizar una educación inclusiva, equitativa y de calidad y promover oportunidades de aprendizaje durante toda la vida para todos" una meta que la educación inclusiva lleva décadas persiguiendo. Podemos decir, por tanto, que se convierte en un marco referencial para hacer efectivo este objetivo en las instituciones educativas. Si entendemos la educación como uno de los derechos humanos inalienables, el hecho de que esta sea inclusiva es un atributo que debe acompañarla en toda propuesta. 
La educación inclusiva es una filosofía educativa que surge a finales del siglo XX con la pretensión de mejorar el acceso, la permanencia y la participación de todos los estudiantes en las instituciones educativas implicando una nueva reconceptualización de la diversidad (Ruíz, 2019). Actualmente es indiscutible que todas las instituciones educativas, incluidas las universidades deben ser para todos. La idea de que la educación constituye un derecho incuestionable de los seres humanos es casi universal. La educación inclusiva busca inequívocamente la igualdad en educación, el derecho que posee cualquier persona de ser educada junto a sus iguales (Ruiz, 2019). Para ello, las acciones educativas estarán principalmente encaminadas a eliminar las barreras que restringen la plena participación de todo el estudiantado en el proceso formativo. Permitiendo así que los estudiantes desarrollen sus talentos y competencias, facilitando el aprovechamiento de beneficios que brinda la sociedad para hacer visible el ejercicio de otros derechos (SENPLADES, 2013). El respeto y el reconocimiento de todas las diferencias presentes en los distintos niveles del sistema educativo, compromete a combatir las desigualdades y la discriminación.

La UNESCO (2006), citado por Calvo y Verdugo (2012), entiende a la educación como un factor de unión si ella considera la diversidad de los individuos y de los grupos humanos y, al mismo tiempo, evita ser una causa que dé lugar a la exclusión social. La educación inclusiva presenta un sistema de determinados valores que se acoge para encaminar el proceso de enseñar y aprender (Colmenero, 2015). Admite una perspectiva que permita al sistema flexibilizar sus planteamientos y ofrecer distintas opciones a los estudiantes diversos. En esta dirección, las escuelas y universidades procurarán garantizar no solo el registro de matrícula de los estudiantes sino mejorar las circunstancias de los ambientes educativos, facilitar la acogida, la permanencia y culminación de sus estudios.

Para Ainscow et al. (2006) la educación inclusiva es un proceso sistemático que deben enfrentar las instituciones educativas que tiene como finalidad eliminar las barreras que limitan la participación. Entre esas barreras se encuentran la voluntad y las competencias para respetar, entender, aceptar y apoyar la diversidad por parte de todos los actores educativos. En este sentido, la educación inclusiva se relaciona con el proceso de reconocer y respetar la diversidad, como elemento previo para favorecer la contribución de todas las personas en la sociedad, escuela o universidad, intentando eliminar todo tipo de situaciones que den paso a la exclusión (Gutiérrez et al., 2018). La educación inclusiva necesita la identificación y eliminación de barreras, poniendo especial atención a las que implican a las personas que se hallan en riesgo de ser excluidos del sistema educativo (Darreche et al., 2010).

Hablar de educación superior inclusiva implica concebir la universidad desde una perspectiva de esperanza y cambio; conlleva la búsqueda y construcción de posibilidades sociales al prescindir las diferencias que muchas veces se perciben como divergencias en el contexto universitario (Gil \& Morales, 2019). Para la universidad constituye un compromiso ético ver la diversidad como una fuente de enriquecimiento (Gallegos, 2015; Herdoíza, 2015), prestando una atención especial a los colectivos o personas con mayor riesgo de exclusión (Barrio de la Puente, 2008). La finalidad de la educación inclusiva está vinculada con la trasformación profunda del sistema educativo al permitir el incremento de la participación de todos los miembros de la comunidad educativa y reducir la exclusión. Hablar de inclusión a nivel universitario conlleva restructurar la cultura, las políticas y las prácticas para que puedan atender a la diversidad.

Para alcanzar el objetivo de la Agenda 2030 de no dejar a nadie atrás, la universidad debe realizar un proceso de análisis y evaluación de sus prácticas, políticas, culturas inclusivas para identificar los procesos de inclusiónexclusión y de esta manera valorar las posibles mejoras. Asumir que los valores, políticas, prácticas de la educación deben beneficiar a los integrantes de la comunidad universitaria resulta evidente para todas las personas que pertenecemos a la comunidad educativa. Sin embargo, no siempre se consigue que cada estudiante aprenda al ritmo que le imponen sus condiciones cognitivas, físicas, sociales, culturales o sensoriales. La educación inclusiva es una responsabilidad social que demanda el compromiso y la participación de toda la comunidad universitaria mediante la implicación y el compromiso de sus integrantes en la búsqueda de formas más acertadas de responder a la diversidad, esperando aprender a aprender de la diferencia (Darreche et al., 2010; Gil \& Morales, 2019; Martínez, 2021).

Desde los espacios universitarios existe un interés creciente por generar cultura inclusiva y por desarrollar prácticas inclusivas a través de la creación de políticas y estrategias educativas. Ante esta necesidad, es preciso conocer y reflexionar sobre las prácticas, valores y políticas inclusivas que se evidencian al acceder a una institución, mediante la implicación, la estabilidad, el éxito y la superación de todo el estudiantado al sistema educativo (Arnaiz \& Azorín, 2014; Booth \& Ainscow, 2000; Echeita, 2013).

La Universidad de Cuenca al igual que todas las Institución de Educación Superior (IES) en el siglo XXI, enfrentan un gran y complejo desafío compartido a nivel mundial, conseguir educación sin exclusiones, una universidad para todos, sin ningún tipo de condición, procurando garantizar la igualdad de oportunidades para todos. Actualmente nos encontramos cada vez más con aulas muy diversas, siempre han sido diversas, pero en los últimos años la heterogeneidad es mayor. Por ello la Universidad de Cuenca intenta acoger los principios de la inclusión y se encuentran adaptando prácticas y modelos educativos que busquen garantizar el aprendizaje de Todos. La inclusión educativa en el terreno de la universidad suscita la incorporación de estudiantes de diversos sectores, asegurando su permanencia, egreso y culminación de su carrera profesional (Universidad de Cuenca, 2019). Se garantiza la participación, el bienestar estudiantil a través de apoyos financieros y educativos, promoviendo espacios de equidad, activando políticas, reformas y apoyos que permitan responder a la diversidad.

A más de estas acciones, en cuanto a la normativa que regula la Educación Superior (ES) y concretamente las políticas que regulan el proceso de admisión, permanencia y culminación de los estudios de todo el estudiantado, encontramos el estatuto actualizado, el Instructivo para garantizar la educación inclusiva, las políticas de acción afirmativa, el plan de mejoras para la inclusión social 2015-2017, la Agenda de Igualdad de Oportunidades entre otras acciones enmarcados dentro de la política y normativa institucional que pretende generar bienestar de 
la comunidad universitaria a nivel de atención a la diversidad y superación de las inequidades que aún persisten dentro de la institución superior (Universidad de Cuenca, 2019).

Teniendo presente este marco normativo que pretende garantizar el aprendizaje y la participación de todos, buscando desde la universidad aportar en la construcción de una sociedad más justa, más incluyente, una sociedad con mayores oportunidades para todos, se ha planteado como principal objetivo del presente estudio: describir las culturas, políticas y prácticas inclusivas dentro de la Universidad de Cuenca, desde la percepción de docentes. Objetivo que parte de la consideración de que, para que la educación inclusiva se vuelva realidad y no quede solo en declaraciones se deberá evaluar estas tres dimensiones, ya que permite determinar si la comunidad universitaria se siente acogida, si sus integrantes se identifican con la institución y cuentan con políticas que respeten la diversidad, así como también si se hace lo posible por desarrollar prácticas que permitan combatir la exclusión, estableciendo facultades accesibles, equitativas y de calidad para todos.

\section{MATERIALES Y MÉTODOS}

\subsection{Instrumento}

Para conocer la situación de las universidades en cuanto a la educación inclusiva es menester valorar las dimensiones: creación de culturas, elaboración de políticas y desarrollo de prácticas inclusivas. Al hacerlo es preciso valorar la experiencia de los distintos miembros de la comunidad universitaria. Con este objetivo se ha diseñado una investigación que cuenta con distintas fases y pretende abarcar a los distintos actores universitarios (estudiantes, docentes y gestores) de la Universidad de Cuenca (Ecuador). En este artículo, nos centraremos en la fase de trabajo con docentes, actualmente 1,153 profesores, que imparten docencia en 12 Facultades.

Para esta fase se ha utilizado el cuestionario Index for Inclusion instrumento original de Booth y Ainscow (2002), en la adaptación a la ES realizada por Salceda e Ibáñez (2015). Para configurar la muestra se llevó a cabo un muestreo probabilístico estratificado (Morales, 2012) obteniendo una muestra de 428 docentes que presenta un $95 \%$ de confianza, con un $3.8 \%$ de error y $50 \%$ de heterogeneidad. La participación en la investigación era anónima, totalmente voluntaria y los docentes debían dar su consentimiento informado. Una vez que contamos con la autorización de los señores decanos de cada facultad, el cuestionario se aplicó en el mes de junio del 2019, de manera presencial, consiguiendo un porcentaje de respuesta del $100 \%$.

El Index for Inclusion, por su diseño, permite evaluar las dimensiones que garantizan la educación inclusiva en los diferentes escenarios educativos a través de seis secciones contenidas en las dimensiones: culturas, políticas y prácticas inclusivas. Este instrumento es uno de los primeros diseñados para apoyar a las escuelas en sus procesos hacia la educación inclusiva. A nivel universitario surge la propuesta de Salceda e Ibáñez (2015), con la finalidad de evaluar culturas políticas y prácticas a nivel de ES para implementar medidas de desarrollo inclusiva para cada una de las dimensiones propuestas en el Índex, situación que posibilitaría el paso de la improvisación a la transformación educativa.

El instrumento consta de 48 ítems y presenta cuatro alternativas de respuesta para cada ítem, las tres corresponden a una escala $(1=$ En desacuerdo, $2=$ Bastante de acuerdo, 3 = Totalmente de acuerdo) siendo la 4 una opción que señala la ausencia de conocimiento sobre el ítem. Además, incorpora un conjunto de elementos diseñados para apoyar a las universidades en el proceso de caminar hacia la meta de convertirse en instituciones educativas con un modelo de educación inclusiva.

Las dimensiones que propone el Index for inclusion adaptado a la educación superior son las siguientes:

Dimensión A: Crear Culturas Inclusivas. - La primera dimensión se relaciona con la formación de un sistema educativo acogedor, colaborador y alentador, en el que cada estudiante sea valorado y se proporcione mayores oportunidades para aportar al aprendizaje y la participación. Contempla dos secciones:

- Sección A.1. Construir Comunidad

- Sección A.2. Establecer valores inclusivos

Dimensión B: Elaborar Políticas Inclusivas. - Pretende legitimar que la inclusión sea el centro del desarrollo de la institución educativa generando políticas que den respuesta a la diversidad. La inclusión educativa es considerada como el foco de cambio, política con la que todos los actores de la comunidad universitaria están comprometidos. De igual manera esta dimensión contiene dos secciones:

- Sección B.1. Desarrollar una Universidad para todas las personas

- Sección B.2. Organizar el apoyo para atender a la diversidad

Dimensión C: Desarrollar prácticas inclusivas. - Cuando se habla de prácticas inclusivas nos referimos a que todas las actividades realizadas en el contexto universitario promueven la participación y el aprendizaje de todo el estudiantado. Las prácticas educativas exhiben las políticas inclusivas y la cultura de la institución. Pretenden, además superar las barreras que en algunos casos aún son visibles. Para evaluar esta dimensión el instrumento cuenta con dos secciones:

- Sección C.1. Orquestar el proceso educativo

- Sección C.2. Movilizar recursos (Salceda \& Ibáñez, 2015)

A través de las tres dimensiones, el Index puede ser utilizado de diferentes maneras adaptándose a las necesidades del contexto donde se aplica.

\subsection{Análisis de los datos}

Los datos fueron procesados y examinados con el software SPSS 24, para obtener los resultados se emplea estadística descriptiva e inferencial. Las frecuencias y porcentajes, las medias y desviaciones estándar de los ítems del instrumento constituyen los estadísticos descriptivos. Se analizó la distribución de frecuencias para las tres dimensiones, así como también la distribución de frecuencias para cada una de las dimensiones y secciones del cuestionario, en relación con resultados obtenidos en los datos sociodemográficos.

El análisis de datos se presenta mediante medidas de tendencia central y dispersión; se empleó la prueba 
estadística T-Student para muestras independientes para la comparación de 2 grupos y ANOVA de un factor para la comparación entre grupos con el post-hoc test de Tukey.

\subsection{Participantes}

Respecto a la caracterización de la muestra, encontramos que la población de estudio lo conformaron 428 docentes de la Universidad de Cuenca. El 54.4\% eran de género masculino, $43.9 \%$ de género femenino y $0.2 \%$ manifestaron no reconocerse en ninguna de estas dos categorías. De la muestra, el 3.5\% de docentes afirmaron tener una discapacidad. Las edades de los docentes oscilaban entre 24 y 65 años $(\mathrm{M}=42.2 ; \mathrm{DE}=8.8)$; en su mayoría se identifican en la etnia mestiza $(83.6 \%$ de docentes). Con respecto a nivel de formación, el $68.9 \%$ contaba con maestría, el $5.4 \%$ con tercer nivel, $16.4 \%$ con doctorado y el $2.6 \%$ con postdoctorado. Especialmente relevante encontramos el dato de que el $40.4 \%$ tenía alguna formación vinculada al tema de la educación inclusiva, consultados sobre la modalidad de formación, el $45.6 \%$ había asistido a charlas, el $29.7 \%$ cursos y el $23.2 \%$ talleres sobre el tema. Conviene indicar que al indagar sobre si hacía falta procesos de formación, el 53\% considera la necesidad de formarse en inclusión educativa y atención a la diversidad. En lo referido a su situación laboral, el $53.4 \%$ era ocasional, $4.3 \%$ técnicos docentes y el $42.3 \%$ titulares. Respecto al área de conocimiento un $25.9 \%$ de docentes corresponden al Área Social, seguido por un $25 \%$ de Ciencias Médicas y un $22.2 \%$ del área de Humanidades, un $18.7 \%$ de Ingeniería y tecnología, mientras que un $8.2 \%$ correspondió al área de Ciencias Agropecuarias (ver Tabla 1).

\section{RESULTADOS}

En este apartado del artículo se presentan los resultados referidos a las dimensiones del Index for Inclusion adaptado a la educación superior, esto es, creación de culturas inclusivas, elaboración de políticas inclusivas y desarrollo de prácticas inclusivas desde la percepción de docentes dentro de la Universidad de Cuenca. Los resultados se desarrollarán en función de las dimensiones y secciones que contempla el Index, de igual manera se explorará la percepción considerando los indicadores, ítems que permitirán contar con puntos de reflexión para analizar los procesos de inclusión educativa dentro de la universidad.

De los resultados visibles en la Tabla 1, llama la atención los datos expresados por los docentes; encontramos que no todos los docentes contestan a las preguntas de este bloque. Es especialmente significativo en las preguntas referidas a la identificación étnica y el hecho de presentar una discapacidad, donde solo responden el $96.7 \%$ y el $90.4 \%$, respectivamente. En estos dos aspectos existe una importante ausencia de respuesta en relación con las otras preguntas referidas a la identificación con la carrera y las áreas de conocimiento. Esta cuestión permite suponer que especialmente la pregunta sobre si tiene o no se tiene discapacidad pudo causar cierto conflicto, puesto que los docentes no se identifican en su totalidad o les cuesta realizar el reconocimiento. Este hecho nos puede llevar a pensar que la discapacidad todavía implica una connotación negativa por lo que, desde identificarse con la misma o identificarse con un grupo étnico minoritario ya se relacionaría con grupos de exclusión. Lo que no sucede con la identificación de la carrera o área de conocimiento que en este estudio se presenta al $100 \%$ la tasa de respuesta.

De estos resultados obtenidos una de las implicaciones que deberá trabajar la universidad en sus intentos de avanzar hacia la construcción de una institución inclusiva será apostar por la sensibilización y la formación social respecto no solo al reconocimiento de la diversidad, sino que la identificación y la valoración del otro, del diferente, de lo diverso como características fundamentales de la sociedad actual.

Los resultados obtenidos en la investigación se han organizado en torno a las tres dimensiones, tal como se ha estructurado el Index for Inclusion adaptado a la ES: El desarrollo de culturas, elaboración de políticas y la aplicación de prácticas inclusivas dentro de la Universidad de Cuenca. Cada una de las dimensiones se presentan por frecuencias y porcentajes con la finalidad de determinar el comportamiento general de los docentes.

Tabla 1. Perfil de los docentes que participaron en la investigación.

\begin{tabular}{llc}
\hline Característica & & Profesores \% (N =428) \\
\hline \multirow{4}{*}{ Etnia } & Blanco & 11.2 \\
& Mestizo & 83.6 \\
& Mulato & 0.5 \\
& Indígena & 0.7 \\
\hline \multirow{2}{*}{ Discapacidad } & Sin discapacidad & 86.9 \\
& Con discapacidad & 3.5 \\
& Ciencias Agropecuarias & 8.2 \\
& Arquitectura y Urbanismo & 4.7 \\
& Artes & 8.2 \\
\multirow{5}{*}{ Facultad } & 4.9 \\
& Jurisprudencia, Ciencias Políticas y Sociales & 12.1 \\
& Ciencias Económicas y Administrativas & 9.3 \\
& Filosofía, Letras y Ciencias de la Educación & 5.4 \\
& Ciencias de la Hospitalidad & 8.9 \\
& Ingeniería & 20.6 \\
& Ciencias Médicas & 4.4 \\
& Odontología & 3.5 \\
& Psicología & 9.8 \\
\hline
\end{tabular}


Tabla 2. Descripción general de la evaluación.

\begin{tabular}{lcc}
\hline \multirow{2}{*}{ Las dimensiones del Index for inclusion } & \multicolumn{2}{c}{ Profesores } \\
& Media & DE \\
\hline A. Creación culturas inclusivas & $\mathbf{2 . 1 4}$ & $\mathbf{0 . 4 1}$ \\
A.1. Construir comunidad & 2.19 & 0.41 \\
A.2. Establecer valores inclusivos & 2.06 & 0.50 \\
\hline B. Elaboración de políticas inclusivas & 1.93 & 0.52 \\
B.1. Desarrollar una universidad para todas las personas & 1.98 & 0.52 \\
B.2. Organizar el apoyo para atender la diversidad & 1.88 & 0.58 \\
\hline C. Desarrollo de prácticas inclusivas & $\mathbf{2 . 1 3}$ & $\mathbf{0 . 5 0}$ \\
C.1. Organizar el proceso educativo & 2.17 & 0.52 \\
C.2. Movilizar recursos & 2.05 & 0.55 \\
\hline
\end{tabular}

Respecto a estas dimensiones a nivel general encontramos un predominio de creación de culturas inclusivas $(\mathrm{M}=$ 2.05; $\mathrm{DE}=0.40$ ) en una medida muy similar al desarrollo de prácticas inclusivas $(\mathrm{M}=2.02 ; \mathrm{DE}=0.48)$, siendo la elaboración de políticas inclusivas la dimensión menos valorada $(\mathrm{M}=1.87 ; \mathrm{DE}=0.46)$. Estos datos pueden estar relacionados con el hecho de que el profesorado asume que las políticas dependen mayormente de las instancias que regulan el sistema de ES y/o de las autoridades que dirigen la universidad, en tanto que las prácticas y la generación de una cultura inclusiva sí dependen directamente de su labor (ver Tabla 2)

\subsection{Creación de culturas inclusivas}

Esta dimensión se relaciona con la formación de un entorno universitario inclusivo, agradable, participativo y confortante. Un espacio donde el estudiantado se sienta valorado, reconocido y donde se le brinde variadas oportunidades para garantizar su aprendizaje y participación. Los resultados de este apartado están relacionados con las secciones que contempla esta dimensión.

Con relación a la sección A.1. Construir comunidad, los indicadores menos conocidos fueron: A.1.4. 'La facultad involucra a la comunidad universitaria en el diseño de propuestas para la mejora de la convivencia interna', situación que relacionamos en este caso la falta de participación del grupo de docentes en el diseño de herramientas o documentos que permitan mejorar la dinámica inclusiva de cada facultad. Con relación al indicador mejor valorado, encontramos el ítem referido al A.1.8. 'las relaciones entre el profesorado y el alumno se basan en el respeto mutuo' $(\mathrm{M}=2.50 ; \mathrm{DE}=0.55)$, mientras que el indicador con la menor percepción fue A.1.1. 'Todo el mundo se siente acogido' $(\mathrm{M}=1.85$; $\mathrm{DE}$ $=0.69$ ). Este escenario permite vislumbrar buenas relaciones entre docentes y estudiantes, pero al mismo tiempo se percibe que en la universidad o en la facultad no todas las personas tienen garantizada su participación (ver Tabla 3).

Por otra parte, en la sección A.2. referida al establecimiento de valores inclusivos encontramos que los indicadores menos conocidos desde la percepción de los docentes de la Universidad de Cuenca fueron: A.2.4. ' $\mathrm{La}$ facultad implementa acciones para prevenir riesgos psicosociales' ( $81.5 \%$ ), y el A.2.7. 'El profesorado tiene expectativas altas sobre el alumnado' $(\mathrm{M}=2.20$; $\mathrm{DE}=$ 0.64). Quizá el resultado del primer caso corresponda a que no todos los docentes están familiarizados con el término 'riesgos psicosociales' y en el segundo indicador se enfoca a las experiencias que los docentes tienen con sus estudiantes a lo largo de su carrera profesional, las mismas que no siempre se reportan como positivas. En esta misma sección, la percepción del indicador mejor evaluado se refiere a A.2.2. 'La facultad transmite al entorno local que es una institución donde se generan transformaciones que contribuyen a mejorar la sociedad' $(\mathrm{M}=2.16 ; \mathrm{DE}=0.65)$, situación que permite rescatar la percepción que presentan los docentes sobre el compromiso que mantiene la Universidad de Cuenca con el sur del Ecuador como una institución de ES comprometida con el cambio social.

\subsection{Elaborar políticas inclusivas}

Esta dimensión se orienta al aseguramiento de la inclusión como eje del progreso de la universidad. Las IES requiere generar políticas que permitan atender y dar respuesta a la diversidad, estás políticas necesitan ser consultadas, socializadas y construidas con toda la comunidad universitaria, con la finalidad de comprometer con las mismas a todos los actores implicados en la universidad.

En la dimensión B: Generación de políticas inclusivas, que cuenta con dos secciones: B.1. 'Desarrollar una universidad para todas las personas' y B.2. 'Organizar el apoyo para atender la diversidad', se encontró que, los indicadores predominantes en el desarrollo de una universidad para todas las personas fueron B.1.2. ' $\mathrm{La}$ facultad es accesible para todas las personas' $(\mathrm{M}=2.05$; $\mathrm{DE}=0.73)$ y B.1.4. 'El estudiantado que ingresa a la facultad recibe atención que garantiza su preparación para la vida y el mundo laboral' $(\mathrm{M}=2.16 ; \mathrm{DE}=0.67)$. Puede observarse que en los indicadores más valorados encontramos que el ingreso a las facultades está garantizado para el estudiantado, situación que puede respaldarse en que los docentes conocen la política que establece el ingreso a la ES, la misma que no depende de la institución propiamente dicha, ni del actuar de los docentes o las autoridades, más bien las políticas de ingreso están determinadas por instancias de orden superior que regulan las IES y que son las encargadas de establecer el ingreso a las distintas universidades en todo el país.

Los docentes al estar vinculados en este caso a la Universidad de Cuenca son conscientes de que las instancias correspondientes se aseguran de proporcionar el ingreso a los aspirantes en las distintas carreras que oferta la universidad año tras año. Además, en relación a la accesibilidad, conocen las posibilidades que se contempla desde la SENESCYT para garantizar el ingreso, estableciendo desde esta instancia políticas de cuotas para responder a los grupos que históricamente han sido 
Tabla 3. Percepción de los docentes, según las dimensiones del Index.

Dimensión A Crear culturas inclusivas: A.1. Construir comunidad

Ítems

A.1.1. Todo el mundo se siente acogido

A.1.2. Los recursos del entorno local se utilizan para mejorar el aprendizaje

A.1.3. La facultad implementa estrategias para vincularse a redes locales y regionales de política social

A.1.4. La facultad involucra a la comunidad universitaria en el diseño

A.1.5. El aula es un espacio social y educativo de participación de propuestas para la mejora de la convivencia interna

A.1.6. Colaboración entre el profesorado y el personal no docente (administrativo, consejería, limpieza)

A.1.7. El trabajo desarrollado por el personal no docente (administrativo, consejería, limpieza) es conocido y valorado pro-estudiantes y profesorado

A.1.8. Las relaciones entre el profesorado y el alumnado se basan en el respeto mutuo

A.1.9. El alumnado participa en los órganos de representación estudiantil (consejo directivo, junta académica, consejo universitario)

A.1.10. El alumnado se apoya mutuamente para lograr metas educativas

A.1.11. Toda la comunidad universitaria siente orgullo de pertenecer a esta universidad

\section{A.2. Establecer valores inclusivos}

\section{Ítems}

A.2.1. Se identifica con la filosofía, los principios y los objetivos de la educación inclusiva

A.2.2. La facultad transmite al entorno local que es una institución donde se generan transformaciones que contribuyan a mejorar la sociedad

A.2.3. La Facultad impulsa acciones que promueven conductas sociales positivas y la solidaridad en oposición al individualismo y el utilitarismo

A.2.4. La facultad implementa acciones para prevenir riesgos psicosociales

A.2.5. La facultad implementa acciones para disminuir las prácticas discriminatorias

A.2.6. Todos los miembros de la facultad son tratados como personas que desempeñan un rol fundamental para el buen funcionamiento de

la misma

A 2.7. El profesorado tiene expectativas altas sobre el alumnado

A.2.8. El profesorado implementa acciones para eliminar las barreras al aprendizaje y la participación

\begin{tabular}{|c|c|c|c|}
\hline \multicolumn{2}{|c|}{ Tasa de respuesta } & \multirow{2}{*}{ M } & \multirow{2}{*}{$\mathrm{DE}$} \\
\hline $\mathrm{n}$ & $\%$ & & \\
\hline 379 & 88.6 & 1.85 & 0.69 \\
\hline 400 & 93.5 & 2.07 & 0.61 \\
\hline 384 & 89.7 & 2.04 & 0.62 \\
\hline 373 & 87.1 & 1.97 & 0.69 \\
\hline 401 & 93.7 & 2.37 & 0.61 \\
\hline 394 & 92.1 & 2.31 & 0.62 \\
\hline 410 & 95.8 & 2.13 & 0.68 \\
\hline 400 & 93.5 & 2.50 & 0.55 \\
\hline 403 & 94.2 & 2.38 & 0.67 \\
\hline 384 & 89.7 & 2.10 & 0.67 \\
\hline 396 & 92.5 & 2.36 & 0.62 \\
\hline \multicolumn{2}{|c|}{ Tasa de respuesta } & \multirow{2}{*}{ M } & \multirow{2}{*}{$\mathrm{DE}$} \\
\hline $\mathrm{n}$ & $\%$ & & \\
\hline 395 & 92.3 & 1.96 & 0.66 \\
\hline 409 & 95.6 & 2.16 & 0.65 \\
\hline 400 & 93.5 & 2.11 & 0.70 \\
\hline 349 & 81.5 & 1.84 & 0.64 \\
\hline 368 & 86.0 & 1.92 & 0.66 \\
\hline 382 & 89.3 & 2.04 & 0.70 \\
\hline 413 & 96.5 & 2.21 & 0.65 \\
\hline 405 & 94.6 & 2.20 & 0.64 \\
\hline \multicolumn{2}{|c|}{ Tasa de respuesta } & \multirow{2}{*}{ M } & \multirow{2}{*}{$\mathrm{DE}$} \\
\hline $\mathrm{n}$ & $\%$ & & \\
\hline 381 & 89.0 & 1.98 & 0.67 \\
\hline 398 & 89.0 & 2.05 & 0.73 \\
\hline 386 & 89.0 & 1.96 & 0.67 \\
\hline 412 & 89.0 & 2.16 & 0.67 \\
\hline 402 & 89.0 & 1.98 & 0.70 \\
\hline 375 & 89.0 & 1.86 & 0.73 \\
\hline 399 & 89.0 & 1.96 & 0.73 \\
\hline 378 & 89.0 & 1.99 & 0.72 \\
\hline
\end{tabular}


Tabla 3. Dimensiones y percepciones de los docentes (continuación).

\begin{tabular}{|c|c|c|c|c|}
\hline \multirow{2}{*}{$\begin{array}{l}\text { B.2. Organizar el apoyo para atender la diversidad } \\
\text { Ítems }\end{array}$} & \multicolumn{2}{|c|}{ Tasa de respuesta } & \multirow[b]{2}{*}{ M } & \multirow{2}{*}{$\mathrm{DE}$} \\
\hline & $\mathrm{n}$ & $\%$ & & \\
\hline B.2.1. La facultad define las políticas para la atención a la diversidad y responde por su divulgación y cumplimiento & 376 & 89.0 & 1.90 & 0.68 \\
\hline B.2.2. Se promueve la investigación y formación del profesorado en temas relacionados con la educación inclusiva & 367 & 89.0 & 1.81 & 0.75 \\
\hline B.2.3. Existen formas de apoyo pedagógico para el alumnado que lo necesita & 369 & 89.0 & 1.93 & 0.69 \\
\hline B.2.4. Los procedimientos tradicionales de evaluación del alumnado se combinan con otros & 376 & 89.0 & 2.08 & 0.68 \\
\hline B.2.5. El profesorado recibe formación para gestionar la disciplina en el aula & 383 & 89.0 & 1.81 & 0.77 \\
\hline B.2.6. Existen alternativas de enseñanza y tutorización no presencial para el alumnado que lo necesita & 378 & 89.0 & 1.92 & 0.71 \\
\hline B.2.7. Se implementan acciones para detectar las relaciones de abuso de poder o bullying & 365 & 89.0 & 1.77 & 0.77 \\
\hline \multirow{2}{*}{$\begin{array}{l}\text { Dimensión C. Desarrollar prácticas inclusivas: C.1. Organizar el proceso educativo } \\
\text { Ítems }\end{array}$} & \multicolumn{2}{|c|}{ Tasa de respuesta } & \multirow{2}{*}{ M } & \multirow{2}{*}{$\mathrm{DE}$} \\
\hline & $\mathrm{n}$ & $\%$ & & \\
\hline C.1.1. Los contenidos de las materias de estudio están conectados a problemáticas sociales relevantes & 417 & 97.4 & 2.19 & 0.63 \\
\hline C.1.2. Los contenidos de las materias de estudio se adecuan a la diversidad del alumnado & 404 & 94.4 & 2.09 & 0.70 \\
\hline C.1.3. Se organizan grupos de aprendizaje para que todo el alumnado se sienta valorado & 401 & 93.7 & 2.08 & 0.70 \\
\hline C.1.4. En la organización de espacios y tiempos se tiene en cuenta a las personas que conforman la comunidad universitaria & 397 & 92.8 & 2.08 & 0.70 \\
\hline C.1.5. Se implica activamente al alumnado en su propio aprendizaje & 420 & 98.1 & 2.25 & 0.62 \\
\hline C.1.6. El profesorado apoya el aprendizaje y la participación de todo el alumnado & 399 & 93.2 & 2.34 & 0.59 \\
\hline C.1.7. El profesorado planifica, revisa y enseña en colaboración con otros docentes & 408 & 95.3 & 2.23 & 0.67 \\
\hline C.1.8. La evaluación motiva y refleja los logros del alumnado & 407 & 95.1 & 2.17 & 0.67 \\
\hline \multirow{2}{*}{$\begin{array}{l}\text { C.2. Movilizar recursos. } \\
\text { Ítems }\end{array}$} & \multicolumn{2}{|c|}{ Tasa de respuesta } & \multirow{2}{*}{ M } & \multirow{2}{*}{$\mathrm{DE}$} \\
\hline & $\mathrm{n}$ & $\%$ & & \\
\hline C.2.1. Se conocen y se aprovechan los recursos de la comunidad universitaria para asegurar la inclusión efectiva de todos sus miembros & 367 & 85.7 & 1.94 & 0.66 \\
\hline C.2.2. Los recursos de la facultad se distribuyen de forma justa para apoyar la inclusión & 352 & 82.2 & 1.94 & 0.71 \\
\hline $\begin{array}{l}\text { C.2.3. La tutoría universitaria es un ámbito integrado en el servicio de orientación de la facultad a disposición de cualquier miembro de la } \\
\text { misma }\end{array}$ & 378 & 88.3 & 2.02 & 0.68 \\
\hline C.2.4. La experiencia del profesorado se aprovecha plenamente para enriquecer el proceso educativo & 407 & 95.1 & 2.11 & 0.67 \\
\hline C.2.5. El profesorado genera recursos para apoyar el aprendizaje y la participación & 409 & 95.6 & 2.22 & 0.65 \\
\hline C.2.6. La diversidad del alumnado se utiliza como recurso para enriquecer el proceso educativo & 397 & 92.8 & 2.13 & 0.71 \\
\hline
\end{tabular}


excluidos del sistema de ES y a aquellos que han presentado menor posibilidad de ingresar como los grupos minoritarios y sectores más vulnerables del país.

En la sección B.2. 'Organizar el apoyo para atender la diversidad', desde la precepción docente resultó la sección con mayores falencias dentro de la Universidad de Cuenca, encontrándose en todos los casos puntuaciones por debajo del punto medio de la escala $(\mathrm{M}=2)$. La excepción la constituye el ítem B.2.4. 'los procedimientos tradicionales de evaluación del alumnado se combinan con otros' $(\mathrm{M}=2.08$; $\mathrm{DE}=0.68)$. La percepción menor obtenida en esta sección se refiere principalmente alindicador B.2.7. concerniente a 'la implementación de acciones para detectar las relaciones de abuso de poder o bullying $(\mathrm{M}=1.77 ; \mathrm{DE}=0.77)$ (ver Tabla 3$)$. En tanto que en la sección B.1. el indicador menos valorado corresponde al B.1.6. 'los nombramientos y las promociones docentes son justas' $(\mathrm{M}=1.86 ; \mathrm{DE}=0.73)$.

$\mathrm{Al}$ respecto en función de los resultados se puede suponer que el profesorado no desarrolla gestiones que permita detectar relaciones de abuso de poder quizá por considerar que en el trabajo con estudiantes a nivel universitario no se requiere o estas relaciones no son visibles, como sí se producen o son mayormente manifiestos en los niveles de educación básica o bachillerato en relación con el acoso escolar.

En el indicador B.1.6. 'el profesorado percibe que los nombramientos y promociones no son justas', este resultado puede explicarse teniendo en cuenta que la promoción dentro de la Universidad de Cuenca exige varios aspectos vinculados a formación, investigación y publicación, elementos que están contemplados en el Reglamento de Escalafón Docente del profesor universitario. Debemos considerar adicionalmente que un alto porcentaje de participantes (exactamente el 53.4\%), se vinculan a tareas de investigación solo ocasionalmente. Este porcentaje supera al $42.3 \%$ en el colectivo de docentes titulares.

\subsection{Desarrollar prácticas inclusivas}

A nivel universitario, desarrollar prácticas inclusivas comprende promover la participación y el aprendizaje de todo el estudiantado en todas las actividades programadas tanto curriculares como extracurriculares por ejemplo, organización de grupos, adecuación de contenidos, organizar espacios y tiempos de acuerdo a la diversidad, desarrollo de la motivación y evaluación en función de logros, generación y aprovechamiento de recursos, desarrollo de tutorías y apoyo psicopedagógico; generalmente las prácticas reflejan la cultura y las políticas de la institución y pretenden superar las barreras que limitan la participación y el aprendizaje de todos.

Vale la pena destacar que en la Dimensión C: Desarrollo de prácticas inclusivas se presentó una tasa de conocimiento superior al $92.8 \%$, a partir de aquí, es preciso suponer que los docentes perciben que las prácticas inclusivas están directamente vinculadas a su actuar pedagógico, más que en la elaboración de políticas o la creación de culturas. En este sentido, los docentes que participan en este estudio reconocen en mayor medida indicadores vinculados a su tarea educativa, es así como en la sección C.1. 'Organizar el proceso educativo', en el indicador C.1.6. 'el profesorado apoya el aprendizaje y la participación de todo el alumnado’ $(\mathrm{M}=2.34 ; \mathrm{DE}=0.59)$, obtienen una puntuación mayor en relación con las demás secciones evaluadas. En esta sección el indicador más débil se refiere a 'la organización de grupos de aprendizaje para que todo el mundo se sienta valorado' $(\mathrm{M}=1.87$; $\mathrm{DE}$ $=0.70)$. De este indicador es posible suponer que varios docentes no están de acuerdo con el trabajo de grupos como una forma de garantizar el aprendizaje, sino que desarrollan otras modalidades de estudio que no están vinculados directamente con esta práctica.

En la segunda sección, referida a movilización de recursos, desde la apreciación de los docentes, sobresalió el ítem 'el profesorado genera recursos para apoyar el aprendizaje y la participación' ( $\mathrm{M}=2.22 ; \mathrm{DE}=0.65)$, seguido del ítem 'la experiencia del profesorado se aprovecha para enriquecer el proceso educativo' $(\mathrm{M}=$ 2.11). Estos datos refuerzan la idea expresada anteriormente, de que el profesorado presenta un mayor compromiso cuando está de por medio su accionar, evidenciándose una mayor relación y por tanto más responsabilidad en los indicadores vinculados a la práctica educativa diaria. Por el contrario, la debilidad más profunda dentro de la sección C.2. movilizar recursos, se vincula con el ítem 'los recursos de la facultad de distribuyen de forma justa para apoyar la inclusión' $(\mathrm{M}=$ 1.94; $\mathrm{DE}=0.71$ ). Nuevamente se puede observar que el manejo de recursos al no ser de responsabilidad del docente determina que los mismos puntúen bajo en la misma, ya sea porque no conocen como se distribuye o porque consideran que la forma como se manejan los recursos no es la adecuada (ver Tabla 3).

En relación con el género, etnia, nivel de instrucción y condición de discapacidad de los docentes no presentaron diferencias significativas en las secciones contempladas en cada una de las dimensiones del cuestionario (ver Tabla 4). En general la formación del docente, las relaciones con los estudiantes, así como el reconocimiento de la diversidad son indicadores reconocidos como un recurso para enriquecer el proceso educativo y avanzar hacia facultades más inclusivas.

La situación laboral del profesorado (docentes ocasionales o docentes titulares) presenta diferencias a la hora de valorar las dimensiones (ver Tabla 5). Los docentes ocasionales consideran que existe mayor nivel de inclusión en las tres dimensiones evaluadas. Encontramos una mayor diferencia en la sección movilizar recursos, donde la valoración de los docentes ocasionales fue mayor. Esta situación que puede deberse a que por su condición de ocasionales perciben que los recursos, los equipos y espacios de práctica son accesibles y pertinentes para llevar adelante una educación inclusiva. Además, pueden tener reticencias al cuestionar que la institución no desarrolle acciones específicas para caminar en la edificación de una cultura inclusiva. Es decir, por los resultados obtenidos, su condición de ocasionales constituye un desencadenante que determina la apreciación positiva con relación al conocimiento y aprovechamiento de los recursos que maneja la universidad para asegurar la inclusión de todos sus miembros.

Finalmente, si consideramos el área de pertenencia de los docentes, de acuerdo con los datos obtenidos, en las secciones: B.1. 'Desarrollar una universidad para todas las personas', B.2. 'Organizar el apoyo para atender la diversidad' y C.1. 'Organizar el proceso educativo' los docentes del área de Ciencias Médicas y de la salud presentaban una percepción significativamente más alta que la de los docentes de las Ciencias Sociales 
Tabla 4. Evaluación según género, etnia y discapacidad (docentes).

\begin{tabular}{|c|c|c|c|c|c|c|c|c|c|c|c|c|}
\hline \multirow{3}{*}{ Sección } & \multicolumn{4}{|c|}{ Género } & \multicolumn{4}{|c|}{ Etnia } & \multicolumn{4}{|c|}{ Discapacidad } \\
\hline & \multicolumn{2}{|c|}{ Masculino } & \multicolumn{2}{|c|}{ Femenino } & \multicolumn{2}{|c|}{ Mestizos } & \multicolumn{2}{|c|}{ Otros } & \multicolumn{2}{|c|}{ No } & \multicolumn{2}{|c|}{ Sí } \\
\hline & $\mathrm{M}$ & $\mathrm{DE}$ & M & $\mathrm{DE}$ & $\mathrm{M}$ & $\mathrm{DE}$ & $\mathrm{M}$ & $\mathrm{DE}$ & $\mathrm{M}$ & $\mathrm{DE}$ & $\mathrm{M}$ & $\mathrm{DE}$ \\
\hline A.1. Construir comunidad & 2.21 & 0.39 & 2.16 & 0.43 & 2.19 & 0.41 & 2.19 & 0.39 & 2.20 & 0.40 & 2.02 & 0.56 \\
\hline A.2. Establecer valores inclusivos & 2.08 & 0.49 & 2.02 & 0.51 & 2.06 & 0.50 & 2.05 & 0.46 & 2.05 & 0.50 & 1.88 & 0.54 \\
\hline B.1. Desarrollar una universidad para todas las personas & 2.00 & 0.51 & 1.93 & 0.51 & 1.97 & 0.51 & 1.98 & 0.53 & 1.96 & 0.51 & 1.79 & 0.58 \\
\hline B.2. Organizar el apoyo para atender a la diversidad & 1.93 & 0.59 & 1.82 & 0.56 & 1.87 & 0.57 & 1.93 & 0.60 & 1.87 & 0.58 & 1.85 & 0.59 \\
\hline C.1. Organizar el proceso educativo & 2.19 & 0.50 & 2.14 & 0.53 & 2.17 & 0.52 & 2.16 & 0.50 & 2.18 & 0.51 & 2.03 & 0.58 \\
\hline C.2. Movilizar recursos & 2.09 & 0.53 & 2.00 & 0.57 & 2.04 & 0.54 & 2.06 & 0.55 & 2.05 & 0.55 & 1.88 & 0.55 \\
\hline
\end{tabular}

Tabla 5. Evaluación según características laborales de docentes.

\begin{tabular}{|c|c|c|c|c|c|c|c|c|c|c|c|c|c|c|}
\hline \multirow{3}{*}{ Sección } & \multicolumn{8}{|c|}{ Experiencia docente } & \multicolumn{6}{|c|}{ Situación laboral } \\
\hline & \multicolumn{2}{|c|}{ Tercer nivel } & \multicolumn{2}{|c|}{ Maestría } & \multicolumn{2}{|c|}{ Doctorado } & \multicolumn{2}{|c|}{ Post doctorado } & \multicolumn{2}{|c|}{ Titular } & \multicolumn{2}{|c|}{ Ocasional } & \multicolumn{2}{|c|}{ Técnico docente } \\
\hline & $\mathrm{M}$ & $\mathrm{DE}$ & $\mathrm{M}$ & $\mathrm{DE}$ & $\mathrm{M}$ & $\mathrm{DE}$ & $\mathrm{M}$ & $\mathrm{DE}$ & $\mathrm{M}$ & $\mathrm{DE}$ & $\mathrm{M}$ & $\mathrm{DE}$ & $\mathrm{M}$ & $\mathrm{DE}$ \\
\hline A.1. Construir comunidad & 2.10 & 0.48 & 2.20 & 0.41 & 2.20 & 0.40 & 2.20 & 0.44 & 2.18 & 0.39 & 2.22 & 0.41 & 2.12 & 0.51 \\
\hline A.2. Establecer valores inclusivos & 1.99 & 0.47 & 2.08 & 0.51 & 2.00 & 0.45 & 2.21 & 0.52 & 2.01 & 0.48 & 2.12 & 0.51 & 1.95 & 0.56 \\
\hline $\begin{array}{l}\text { B.1. Desarrollar una universidad para todas las } \\
\text { personas }\end{array}$ & 1.97 & 0.54 & 2.00 & 0.52 & 1.92 & 0.49 & 2.15 & 0.57 & 1.94 & 0.50 & 2.01 & 0.52 & 1.84 & 0.63 \\
\hline $\begin{array}{l}\text { B.2. Organizar el apoyo para atender a la } \\
\text { diversidad }\end{array}$ & 1.73 & 0.60 & 1.91 & 0.59 & 1.90 & 0.56 & 2.04 & 0.51 & 1.87 & 0.59 & 1.91 & 0.57 & 1.87 & 0.61 \\
\hline C.1. Organizar el proceso educativo & 1.99 & 0.61 & 2.20 & 0.52 & 2.18 & 0.51 & 2.25 & 0.49 & 2.12 & 0.52 & 2.23 & 0.50 & 2.19 & 0.55 \\
\hline C.2. Movilizar recursos & 1.99 & 0.62 & 2.08 & 0.54 & 2.01 & 0.53 & 2.23 & 0.54 & 1.97 & 0.53 & 2.12 & 0.55 & 2.06 & 0.68 \\
\hline
\end{tabular}

Tabla 6. Comparaciones de medias de columna según área de conocimiento (docentes).

\begin{tabular}{|c|c|c|c|c|c|c|c|c|c|c|}
\hline \multirow[t]{2}{*}{ Sección } & \multicolumn{2}{|c|}{ Ingeniería y Tecnología } & \multicolumn{2}{|c|}{$\begin{array}{l}\text { Ciencias Médicas y la } \\
\text { Salud }\end{array}$} & \multicolumn{2}{|c|}{ Ciencias Agropecuarias } & \multicolumn{2}{|c|}{ Ciencias Sociales } & \multicolumn{2}{|c|}{$\begin{array}{l}\text { Ciencias de las } \\
\text { Humanidades }\end{array}$} \\
\hline & $\mathrm{M}$ & $\mathrm{DE}$ & $\mathrm{M}$ & $\mathrm{DE}$ & $\mathrm{M}$ & $\mathrm{DE}$ & $\mathrm{M}$ & $\mathrm{DE}$ & $\mathrm{M}$ & $\mathrm{DE}$ \\
\hline A.1. Construir comunidad & 2.21 & 0.35 & 2.25 & 0.39 & 2.24 & 0.50 & 2.13 & 0.41 & 2.17 & 0.44 \\
\hline A.2. Establecer valores inclusivos & 2.12 & 0.47 & 2.14 & 0.47 & 2.08 & 0.60 & 1.97 & 0.46 & 2.03 & 0.54 \\
\hline $\begin{array}{l}\text { B.1. Desarrollar una universidad para todas las } \\
\text { personas }\end{array}$ & 1.96 & 0.48 & 2.11 & 0.51 & 2.02 & 0.63 & 1.90 & 0.48 & 1.92 & 0.53 \\
\hline $\begin{array}{l}\text { B.2. Organizar el apoyo para atender a la } \\
\text { diversidad }\end{array}$ & 1.85 & 0.54 & 2.07 & 0.56 & 1.92 & 0.63 & 1.76 & 0.55 & 1.83 & 0.60 \\
\hline C.1. Organizar el proceso educativo & 2.10 & 0.46 & 2.32 & 0.53 & 2.22 & 0.54 & 2.04 & 0.52 & 2.20 & 0.49 \\
\hline C.2. Movilizar recursos & 2.03 & 0.54 & 2.15 & 0.52 & 2.13 & 0.60 & 1.94 & 0.53 & 2.06 & 0.58 \\
\hline
\end{tabular}


(ver Tabla 6). Conviene también indicar que los docentes de esta área presentaron en la sección B.2. puntuaciones significativamente mayores que el área de Humanidades.

En la sección C.1. 'Organizar el proceso educativo' el área de Ciencias Médicas obtuvo también una percepción más alta que el área de Ingeniería y Tecnología. Lo obtenido permite exponer que, desde la percepción de los docentes, el área de Ciencias Médicas en cuanto a las dimensiones del Index for Inclusion avanzan a la construcción de una cultura inclusiva, por sobre los docentes de las áreas de Sociales y sobre todo de las áreas técnicas y de las ingenierías, que mantienen una percepción menor sobre las dimensiones de la educación inclusiva evaluadas en esta investigación.

En relación con la percepción de los docentes por facultad se puede indicar que, en base a los resultados encontrados, los docentes de la facultad de Ingeniería eran quienes otorgaban la calificación más baja en la mayoría de las secciones del Índex. Suponemos que estos resultados pueden deberse a que los docentes de esta facultad al ofertar carreras que se fundamentan en la ciencia y la tecnología se enfocan a una mayor preparación científicotécnica y no se acentúan elementos vinculados a la inclusión.

De igual manera los resultados reflejan que la facultad de Arquitectura presentó puntuaciones significativamente más altas en las secciones: C.1. 'organizar el proceso educativo' y C.2. 'movilización de recursos' que el área de Economía y en la sección C.2. 'movilización de recursos' una percepción mayor que el área de Hospitalidad. Secciones que corresponden a la dimensión prácticas inclusivas, vinculados con el perfil de estos profesionales. En esta línea Méndez et al. (2014) en su artículo: "Arquitectura y Urbanismos ¿inclusivos?" sostienen, que la formación en educación inclusiva está vinculada con la práctica pues los profesionales de esta área necesitan diseñar ciudades inclusivas y sustentables donde se favorezca el intercambio entre individuos, culturas, géneros, generaciones o grupos sociales.

\section{DISCUSIÓN}

La revisión de la literatura permite evidenciar el interés por la inclusión educativa en el contexto de la ES, en los últimos años varios estudios tienen como punto de partida el Índice de inclusión. Esta guía ofrece a las instituciones educativas la posibilidad de mejorar, a través de la evaluación de tres dimensiones relacionadas: culturas, políticas y prácticas inclusivas (Rodríguez et al., 2020). Con los resultados del presente estudio utilizando el Index for Inclusion, podemos presumir que la educación inclusiva no implica solo la reducción de barreras que restringen el aprendizaje y la participación, sino conlleva el reconocimiento de los docentes como los principales actores del proceso educativo, quienes a través de su labor pueden limitar o posibilitar la igualdad de oportunidades y la participación plena de todo el estudiantado con el objetivo de conseguir una educación eficaz e incluyente.

A nivel de normativa existen algunos lineamientos que orientan a la ES a trabajar hacia modelos de educación inclusiva (LOES, 2010), de allí que resulta importante conocer la percepción de los docentes como uno de los actores clave del contexto universitario, su apreciación sobre la construcción de políticas, la aplicación de prácticas y la creación de culturas inclusivas resulta fundamental para avanzar hacia sistemas de educación inclusiva. En este artículo mostramos los resultados conseguidos tras la aplicación del Index for Inclusion, datos que posibilitan a la Universidad de Cuenca construir un análisis sobre las primeras barreras con las que deben confrontar los estudiantes al acceder a este espacio universitario.

Los resultados alcanzados en las tres dimensiones permiten poner de relieve algunas coyunturas y retos a los que se enfrenta la universidad para transformar y crear un ambiente más inclusivo. Se identifica también las estrategias y buenas prácticas como: adecuar los contenidos de estudio a la diversidad, involucrar a los estudiantes en su propio aprendizaje, evaluación motivadora entre otras, que se pueden generar desde los docentes para la eliminación de factores relacionados con la exclusión, es decir, valorar el rol determinante del docente en el avance hacia contextos inclusivos. Coincidimos en este punto con varios estudios que se han enfocado a resaltar la importancia de los docentes como un elemento fundamental para avanzar hacia la educación inclusiva (Arnaiz \& Azorín, 2014; Gonzáles, 2016; Gutiérrez et al., 2018; Hurtado et al., 2019). En esta misma línea, otros estudios fundamentan que la equidad, la inclusión, la participación de todos son condiciones ineludibles de la ES y deben estar promovidas principalmente por los docentes (Heskia et al., 2019). Docentes que conozcan a sus estudiantes y favorezcan el aprendizaje, valoren la diversidad, desarrollen prácticas inclusivas, promuevan una cultura universitaria participativa y sin barreras para el aprendizaje.

De los resultados desde la percepción docente encontramos, que la dimensión más débil se refiere a la elaboración de políticas inclusivas, pese a que la inclusión educativa constituye un tema de política educativa actual, la respuesta a la diversidad, la participación y supresión de barreras contemplados en el enfoque de derechos son elementos poco conocidos desde los indicadores del Index for Inclusion, que pueden medir la inclusión referida a políticas inclusivas. Estos resultados coinciden con otros estudios, por ejemplo, Ruiz (2019) señala que, si bien existen políticas de educación inclusiva planteadas desde el estado, la falta de continuidad de estas constituye una brecha que obstaculiza el avance de la inclusión. De igual manera Cotán (2017) menciona que generalmente en casi todas las universidades existen políticas inclusivas, sin embargo, hacen falta procesos de información y socialización sobre mecanismos de educación inclusiva que se estén desarrollando que, al no ser conocidos, tiene un grado de percepción bajo o en ocasiones es considerado como inexistente.

Teniendo presente estos resultados, creemos que este sería uno de los caminos a transitar desde la Universidad de Cuenca. Las políticas empujan un proceso de innovación continua, y deben incluir un mecanismo de sensibilización hacia el profesorado. El desarrollo de estas acciones facilitaría equilibrar la percepción positiva de las prácticas que presentan con la percepción de la dimensión elaboración de políticas inclusivas.

Uno de los resultados importantes indica que el $40.4 \%$ de docentes participó en alguna formación en educación inclusiva. Podemos pensar que los docentes con formación en educación inclusiva proponen estrategias para ayudar a sus estudiantes a descubrir su potencial de 
aprendizaje, sin establecer barreras, ni reducir expectativas, sino reconociendo la equidad y la inclusión como parte de los procesos educativos. Las investigaciones de Martínez (2021) refuerza este hallazgo, este autor señala que la formación permite comprender y apreciar las distintas perspectivas, respetar los distintos puntos de vista, interactuar de forma respetuosa y desde el respeto de los otros, buscando crear oportunidades para todos con la finalidad de conseguir sociedades más inclusivas. En esta misma línea autores como Hurtado et al. (2019) explican que la formación docente en educación inclusiva contribuye al desarrollo de prácticas inclusivas y que por ello la formación docente deberá ser permanente. Si estas investigaciones se comparan con los resultados obtenidos en el indicador B.2.2. referida a la 'promoción de la investigación y formación en temas relacionados con la educación inclusiva', encontramos que la percepción es menor en relación a los demás indicadores, por lo que, la Universidad de Cuenca deberá apuntar a corregir este indicador si pretende trabajar en la construcción de políticas inclusivas desde la formación docente.

Otro de los elementos consultados tiene que ver con la situación laboral de los docentes, quienes dentro de la Universidad de Cuenca se dividen en titulares, ocasionales y técnicos docentes. Conforme a las dimensiones del Index que fueron consultadas, los docentes ocasionales perciben mayor nivel de inclusión en las tres dimensiones, destacándose sobre todo la sección C.2. 'Movilizar recursos', apartado que se refiere a generar, conocer y aprovechar recursos para enriquecer el proceso educativo, así como también considerar la diversidad como un recurso que enriquece el aprendizaje; al respecto no se encuentran estudios que permita comparar la condición de titularidad o estabilidad laboral de los docentes. Encontrándose más bien investigaciones que avalan la calidad de la formación en inclusión educativa, puesto que, más que la titularidad de los docentes, las limitaciones de la educación se encuentran determinadas por el desconocimiento para trabajar con la diversidad del estudiantado, por lo que, independientemente de su situación laboral la formación de los docentes debe contemplar competencias que posibilite un accionar con la pretensión de lograr que todo el estudiantado pueda desarrollarse más allá de sus diferencias (Hurtado et al., 2019).

Resulta importante mencionar que a nivel de Ecuador no existen estudios que se hayan realizado utilizando el Index for Inclusion adaptado a la ES, (instrumento que fue manejado en esta investigación). Los resultados son discutidos con estudios realizados en ES, pero referidos a procesos de inclusión de estudiantes con discapacidad, investigaciones efectuadas en otros países o investigaciones en educación básica o bachillerato. Estos estudios promueven la importancia del desarrollo de prácticas inclusivas desde el actuar de los docentes y contienen resultados que fortalecen los resultados de esta investigación vinculados con la percepción de prácticas inclusivas por parte del profesorado, sin embargo, hace falta investigar más en el contexto de la ES.

Finalmente, de los resultados derivados en la aplicación del Index, una vez puntualizadas las percepciones de los docentes en torno a las dimensiones consultadas y encontrándose diferencias significativas entre las facultades, es necesario avanzar en un plan que permita a la Universidad de Cuenca caminar hacia la inclusión educativa, esto en consonancia con uno de los objetivos de la herramienta referido al planteamiento de propuestas de mejoramiento e innovación.

\section{CONCLUSIONES}

En el análisis de los resultados sobre las dimensiones del Índex encontramos que existen diferencias reveladoras, destacándose la percepción significativa en lo relacionado al desarrollo de prácticas y creación de culturas por encima de la elaboración de políticas inclusivas, lo que permite suponer que los docentes consultados consideran elementos de atención a la diversidad, comprensión por el otro, inclusión, presencia y participación, aspectos que contribuyen en la construcción de una educación superior inclusiva.

Los docentes parecen vincular una integración de la educación inclusiva desde estrategias bottom up (desde la práctica y la cultura inclusiva) más que top down (desde las políticas). Es una cuestión interesante ya que está presente la formación y la voluntariedad de los equipos, más que una normativa de carácter obligatorio. Sin embargo, debemos tener en cuenta que desde las decisiones de política universitaria puede promoverse medidas que apoyen enérgicamente la educación inclusiva y en este sentido la labor de los gestores para hacerlas visibles es muy importante.

Con respecto a las secciones evaluadas, encontramos que existe un apoyo mayoritario a aquellos elementos que implican acción y toma de decisiones. Se considera que los estudiantes deben estar involucrados en la tarea de avanzar hacia una universidad verdaderamente inclusiva, destacándose el apoyo que los docentes deben facilitar para el aprendizaje y participación de todos.

En ese sentido los valores y las actitudes orientan la práctica y las percepciones positivas hacia el asentimiento de la diversidad, entonces la formación del profesor establecerá su accionar hacia la educación inclusiva en mayor proporción. Es necesario contar con formación y sensibilidad por parte de los docentes ante la diversidad. Encontramos algunas dudas sobre esa formación, al apreciar la falta de respuesta en las preguntas iniciales referidas a auto identificación étnica y discapacidad. Pese a ser un porcentaje menor, no es una cuestión baladí y merecería el diseño de una línea de formación específica para docentes.

Los docentes califican significativamente la necesidad de organizar políticas, desarrollar prácticas y establecer culturas inclusivas dentro de la universidad estando presentes los principales actores académicos, como una exigencia actual en sustento a la construcción de una sociedad inclusiva y de paz. Igualmente, los análisis avalan la pertinencia de elaborar programas de formación docente en la línea de educación inclusiva con el propósito de favorecer a la consecución de los objetivos de la Agenda del 2030 que apunta a la transformación de la ES para que sea franca, inclusiva y eficiente.

Finalmente, el estudio plantea en su análisis prospectivo la posibilidad de ampliar la muestra al grupo de estudiantes de las distintas facultades, así mismo se sugiere aplicar grupos focales o la técnica de la entrevista con actores claves, como son los docentes coordinadores de las facultades con el Departamento de Bienestar 
Universitario con la finalidad de obtener mayores resultados sobre los indicadores referidos a las tres dimensiones que han sido evaluadas en la investigación efectuada dentro de la Universidad de Cuenca.

\section{AGRADECIMIENTO}

Los datos que sustentan esta publicación corresponden a resultados obtenidos dentro del proyecto "Percepción de docentes y estudiantes sobre culturas, políticas y prácticas inclusivas en educación superior". Proyecto ganador del XVII concurso de proyectos de investigación de la Universidad de Cuenca (DIUC). Expresamos nuestra gratitud a docentes de las doce facultades de la universidad que participaron en esta investigación, a las autoridades de las facultades quienes autorizaron la aplicación del instrumento, así como también a Directores de Carrera que facilitaron el estudio en las distintas juntas académicas de carrera.

\section{REFERENCIAS}

Ainscow, M., Booth, T., \& Dyson, A. (2006). Improving schools, developing inclusion. London, UK: Routledge Publisher. https://doi.org/10.4324/9780203967157

Arnaiz, P., \& Azorín, C. (2014). Autoevaluación docente para la mejora de los procesos educativos en escuelas que caminan hacia la inclusión. Revista Colombiana de Educación, 67, 227-245.

Asamblea Nacional. (2010). Ley Orgánica de Educación Superior (LOES). Registro Oficial, Suplemento 298: 183. Quito, Ecuador: Presidencia de la República del Ecuador,

Barrio de la Puente, J. (2008). Hacia una educación inclusiva para todos. Revista Complutense de Educación, 20(1), 13-31.

Booth, T., \& Ainscow, M. (2000). Index for inclusion. $130 \mathrm{pp}$. Bristol, UK: Centre for Studies on Inclusive Education (CSIE).

Booth, T., \& Ainscow, M. (2002). Index for inclusion: developing learning and participation in schools. 106 pp. Bristol, UK: Centre for Studies on Inclusive Education (CSIE).

Calvo, M., \& Verdugo, M. (2012). Educación inclusiva, ¿Una realidad o un ideal? Edetania, 41, 17-30.

Colmenero, M. (2015). Caminando hacia una educación inclusiva. Caminando hacia una sociedad inclusiva. Iniciación a la investigación, 6(4), 1-18.

Cotán, A. (2017). Educación inclusiva en las instituciones de educación superior: narrativas de estudiantes con discapacidad. Revista Española de Discapacidad, 5(I), 43-61. https://doi.org/10.5569/2340-5104.05.01.03

Darreche, L., Fernández, J., \& Goicoechea, P. (2010). Educación inclusiva ¿La educación inclusiva como utopía que nos ayuda a caminar? Buenos Aires, Argentina: Congreso Iberoamericano de Educación METAS 2021, 21 págs.
Echeita, G. (2013). Inclusión y exclusión educativa. De nuevo voz y quebranto. Revista Iberoamericana sobre Calidad, Eficacia y Cambio en Educación, 11(2), 100-118.

Gallegos, M. (2015). La educación inclusiva una respuesta a los postulados del Buen Vivir. En la Educación Inclusiva una Respuesta a los postulados del Buen Vivir. Quito, Ecuador: Editorial Abya-Yala. Recuperado de https://dspace.ups.edu.ec/handle/123456789/11031

Gil, A., \& Morales, M. (2019). Diversidad y educación inclusiva en Universidades: Cambiar estigmas y ordenar conceptos. Revista Metropolitana de Ciencias Aplicadas, 2(1), 160 -165

González, M. (2016). Propuesta de aplicación del “Index for Inclusion". XII Congreso internacional y XXXIII Jornadas de Universidades y Educación Inclusiva. 11 págs. Madrid, España: Universidad de Complutense.

Gutiérrez, M., Martín, M., \& Jenaro, C. (2018). La cultura, pieza clave para avanzar en los centros educativos. Revista de Educación Inclusiva, 11(2), 13-26.

Herdoíza, M. (2015). Construyendo igualdad en la educación superior. Fundamentación y lineamientos para transversalizar los ejes de igualdad y ambiente. 176 págs. Quito, Ecuador: SENESCYT/UNESCO. Recuperado de https://www.educacionsuperior.gob.ec/wpcontent/uploads/downloads/2018/11/Construyendoigualdad-en-la-educacion-superior_nov_2018.pdf

Heskia, M., Lepeley, C., Recabarren, C., \& Suarez, M. (2019). Docencia para la inclusión en Educación Superior. Desarrollando prácticas inclusivas. 78 págs. Universidad del Desarrollo. Recuperado de https://repositorio.udd.cl/bitstream/handle/11447/2797/ Docencia\%20para\%201a\%20inclusión\%20en\%20educ ación\%20superior\%20desarrollando $\% 20$ competencias $\% 20$ inclusivas.pdf?sequence $=1 \&$ is A llowed=y

Hurtado, Y., Mendoza, R., \& Viejó, A. (2019). Los desafíos de la formación docente inclusiva: Perspectivas desde el contexto Latinoamericano. Revista Internacional de Apoyo a la Inclusión, Logopedia, Sociedad y Multiculturalidad. 5(2). https://dx.doi.org/10.17561/riai.v5.n2.9

Martínez, M. J. (2021). Inclusión educativa comparada en Unesco y OCDE desde la cartografía social. Educación XXI, 24(1), 93-115, http://doi.org/10.5944/educXX1.26444

Méndez, S., Saura, M., \& Muntañola, J. (2014). Arquitectura y urbanismo ¿Inclusivos? Conference: Barcelona Inclusiva 2014. 1er Congreso Internacional de Orientación para una Sociedad Inclusiva. Barcelona. http://hdl.handle.net/2117/24191

Morales, P. (2012). Estadística aplicada a las Ciencias Sociales. Tamaño necesario de la muestra. Recuperado de https://web.upcomillas.es/personal/peter/ investigacion/Tama\%floMuestra.pdf

Rodríguez, E., González, F., Pastor, E., \& Vidal, R. (2020). Validación de un cuestionario sobre la actitud docente a la educación inclusiva en Chile. Foro Educacional, 35, 63-68. https://dx.doi.org/10.29344/07180772.35.2650 
R. Clavijo \& M. Bautista: Percepción sobre las culturas institucionales, políticas y prácticas inclusivas

Ruiz, R. (2019). Políticas y prácticas pedagógicas inclusivas para la generación de una cultura inclusiva. 230 págs. Universidad de Tolima, Ibagué, Colombia: Editorial Ibagué.

Salceda, M., \& Ibáñez, A. (2015). Adaptación del Index for Inclusion al ámbito de la educación superior: Estudio preliminar. Intangible Capital, 11(3), 508-545. https://doi.org/10.3926/ic.647

SENPLADES. (2013). Plan Nacional de Desarrollo y Plan Nacional para el Buen Vivir 2009-2013: Construyendo un Estado Plurinacional e Intercultural. ISBN: 978-9978-92-794-6 Secretaría Nacional de Planificación y Desarrollo - SENPLADES. Quito, Ecuador.
UNESCO (2006). La educación inclusiva, el camino hacia el futuro. Conferencia internacional de educación. Centro Internacional de Conferencias Ginebra, UNESCO, ED/BIE/CONFINTED48/1.

UNESCO. (2015). El desarrollo sostenible comienza por la educación. Cómo contribuir la educación a los objetivos propuestos para después de 2015. Organización de las Naciones Unidas para la Educación, la Ciencia y la Cultura. Recuperado de https://www.entreculturas.org/sites/default/files/educac ion_y_ods.pdf

Universidad de Cuenca. (2019). Informe de Gestión 2019. Cuenca, Ecuador: Universidad de Cuenca. 